\title{
Relación entre frecuencia y percepción de uso tecnológico, personalidad, afecto y variables neuropsicológicas en población adulta joven
}

\section{Relationship between frequency and perception of technological use, personality, affect and neuropsychological variables in young adult population}

\author{
Cristina Clavero Blanco, Fernando Gordillo León y Miguel Ángel Pérez Nieto \\ Universidad Camilo José Cela, Campus Villafranca, Madrid, España
}

\begin{abstract}
Resumen
El uso de las tecnologías tiene efectos positivos y negativos sobre los procesos cognitivos y afectivos, que en las últimas décadas han atraído la atención de los investigadores. En el presente estudio se investigó la relación entre la frecuencia del uso tecnológico y su influencia en el control inhibitorio y la capacidad atencional en adultos jóvenes, así como el papel jugado por la personalidad y el estado afectivo. Participaron 90 personas con edades comprendidas entre los 18 y 34 años, que realizaron las siguientes pruebas: Test de Stroop; Trail Making Test; Test Atencional d2; Cuestionario de Uso Problemático de las Nuevas Tecnologías; Eysenck Personality Questionnaire Revised-Abbreviated; y el Cuestionario de Afecto Positivo y Negativo. Los resultados mostraron relación significativa e inversa entre la frecuencia de uso de tecnologías como Internet y la Televisión, y las capacidades de inhibición y atención. Por otro lado, se observó una relación significativa y directa entre el uso de Internet y el Móvil, y los niveles de neuroticismo y afecto negativo. Estos resultados apuntan a un efecto negativo del exceso de uso de determinadas tecnologías sobre las capacidades cognitivas, que podría verse modulado por variables afectivas y de personalidad.
\end{abstract}

Palabras clave: atención; control inhibitorio; estado afectivo; tecnologías; velocidad de procesamiento.

\begin{abstract}
In recent decades, researchers have addressed the use of technologies because of its positive and negative effects on cognitive and affective processes. The present study investigated the relationship between the frequency of use of technologies, its influence on inhibitory control and attention span, and the role played by personality and affective status in a young adult population. The study included 90 participants between the ages of 18 and 34 years. They were administered the following tests: Stroop test; Trail Making Test; D2 Test of Attention; Questionnaire on the Problem Use of New Technologies; Eysenck Personality Questionnaire Revised-Abbreviated; and the Positive and Negative Affect Schedule. A significant inverse relationship was found between the frequency of use of technologies, such as the internet and television, and inhibition and attention capabilities, whereas a significant direct relationship was found between the use of the internet and mobile phones and levels of neuroticism and negative affect. These results suggest that the excessive use of certain technologies may have a negative effect on cognitive abilities that may be modulated by affective and personality variables.
\end{abstract}

Keywords: attention; inhibitory control; affective state; technologies; processing speed.

Cómo citar este artículo: Clavero-Blanco, C., Gordillo-León, F. Y Pérez-Nieto, M. A. (2019). Relación entre frecuencia y percepción de uso tecnológico, personalidad, afecto y variables neuropsicológicas en población adulta joven. Escritos de Psicología, $12,69-80$.

Correspondencia: Cristina Clavero Blanco. Universidad Camilo José Cela. Departamento de Psicología, Castillo de Alarcón n 49. 28692-Villafranca del Castillo (Madrid). E-mail: cristicl@ucm.es. E- mail del coautor Fernando Gordillo León, fgordillo@ ucjc.edu. E-mail del coautor Miguel Ángel Pérez Nieto mperez@ucjc.edu. 


\section{Introducción}

Hace más de diez años, y según la Fundación Pfizer (2009), el 98\% de los jóvenes españoles entre 11-20 años eran usuarios de Internet, de los cuales un 70\% afirma conectarse al menos durante una hora y media al día y entre un 3 y $6 \%$ realizan un uso abusivo (Odriozola, 2012). Asimismo, el Instituto Nacional de Estadística (INE, 2013) afirmaba que el móvil era utilizado en el 96,1\% de las viviendas españolas y el $73,4 \%$ de los domicilios disponían de un ordenador, alcanzando un porcentaje del $69,8 \%$ los hogares con acceso a Internet (Rial, Gómez, Braña y Varela, 2014). En la última década se ha producido un incremento considerable, hasta ascender de un $83,4 \%$ el porcentaje de hogares que disponían de acceso a la Red (INE, 2017) a un 91,4\% en el último año (INE, 2019). Estos datos son característicos de la población de jóvenes adultos que han crecido con el uso de las Tecnologías, en un contexto de hiperconectividad social, siendo, por tanto, una población sensible a desarrollar problemas conductuales y cognitivos, derivados de la excesiva frecuencia de uso de estas tecnologías.

Esta excesiva frecuencia de uso podría derivar en un problema de adicción, caracterizado principalmente tanto por la pérdida de control como por una marcada dependencia. Teniendo esto en cuenta, cualquier conducta normal y/o saludable puede convertirse en una conducta problema cuando se produce un aumento considerable en la frecuencia de uso, la cantidad de tiempo y dinero invertido y el grado en que dicha conducta interfiere con la vida cotidiana (Andreassen, Billieux, Griffiths, Kuss, Demetrovics, Mazzoniy y Pallesen, 2017; Echeburúa, 1999). Sin embargo, aunque algunos autores indiquen que una conducta normal puede convertirse en patológica en base a los criterios de intensidad y frecuencia de uso, dinero invertido y grado de interferencia en las relaciones laborales, sociales y/o familiares (Echeburúa, Corral y Amor, 2005), aún no existe una definición concreta y delimitada sobre los criterios que caracterizan la adicción a las tecnologías (Labrador y Villadangos, 2010). Por ello, Carbonell et al., (2012) señalan que la prudencia de algunos autores a la hora de utilizar la terminología "adicción al móvil" se basa en ciertos factores como son la ausencia de usuarios problemáticos identificados, cuestionarios que pueden medir más "percepción" que adicción, y la tendencia a normalizar dicha conducta y/o los problemas que surgen en la delimitación del tiempo a partir del cual puede considerarse un uso excesivo. A su vez, esta postura es apoyada por estudios que revisan el concepto y los instrumentos de medida utilizados y cuyos resultados concuerdan con la ausencia de evidencia empírica que demuestre la adicción a Internet (véase Van Rooij y Prause, 2014). Atendiendo a estos criterios, resulta una ardua tarea diferenciar el uso excesivo o adicción a Internet de un uso normal, por lo que algunos autores como Beranuy, Chamarro, Graner y Carbonel (2009) proponen emplear la etiqueta "adicción a Internet" cuando se cumplan los criterios del juego patológico, así como los criterios de otras adicciones comportamentales o a sustancias (Castro, Vinaccia, y Ballester-Arnal, 2018), o cuando el uso de Internet genere una constante preocupación, control ineficaz sobre el uso realizado y esté presente la necesidad constante de poder tener acceso a él (Yudes, Rey y Extremera, 2019).

En esta dirección, existe una marcada confusión conceptual que deriva en la ausencia de consenso sobre los principios y criterios que definen la adicción tecnológica, cuya consecuencia es la falta de inclusión de un diagnóstico específico para este tipo de trastornos en los manuales diagnósticos como el DSM IV-TR, DMS-5 y CIE-10 (Rial et al., 2014). A pesar de esto, algunos autores establecen ciertos criterios de carácter general, recogidos del DSM IV-TR, en concreto para el diagnóstico de la adicción al móvil, indicando la necesidad de padecer al menos tres o más de los criterios mencionados durante 12 meses (Billieux et al., 2015), como son, tolerancia, abstinencia, uso más frecuente de lo previsto, uso descontrolado, impacto negativo en el área laboral, social y personal, y consciencia del uso descontrolado. A su vez, el DSM-5 recoge como única adicción comportamental el juego patológico, dentro de la categoría "Trastornos no relacionados con sustancias". Se han encontrado semejanzas conductuales entre el trastorno de juego por Internet (al que se ha calificado también como trastorno por uso de Internet, adicción a Internet o a los juegos) y el juego patológico y el consumo de sustancias, defiendo como característica principal del trastorno de juego por internet una participación constante en los juegos de ordenador durante muchas horas (APA, 2014).

Teniendo en cuenta los criterios mencionados, el DSM-IV TR (APA, 2000) incluye las adicciones comportamentales en los Trastornos del Control de los Impulsos, donde se clasifica el Juego Patológico como diagnóstico independiente quedando el resto de las categorías diagnósticas incluidas en el apartado "Trastornos del control de los impulsos no especificados" (Pedrero-Pérez, Rodríguez-Monje y Ruiz-Sánchez de León, 2012), siendo modificada tal clasificación en el DSM-5, incluyendo el Juego Patológico en la categoría de "Trastornos relacionas con sustancias y adictivos", en la CIE-10 en "Trastornos de los hábitos y del control de los impulsos" y en la CIE-11, dentro de la categoría "Trastornos de consumo de sustancias y conductas adictivas", se han incluido dos conductas adictivas sin sustancia: el Juego Patológico y el "Internet Gaming disorder". Inicialmente, una conducta adictiva está contro- 
lada por la satisfacción conductual experimentada durante el uso o consumo del elemento que causa dicha adicción, convirtiéndose en un reforzador positivo. Sin embargo, este reforzador se transforma, finalmente, en un reforzador negativo que controla la conducta adictiva manifestándose tal control en el alivio de la tensión emocional que deriva del uso o consumo reiterado (Echeburúa, 1999; Marks, 1990; Potenza, 2006; Treuer, Fábian y Füredi, 2001). Por tanto, lo que se observa en un sujeto adicto es la aparición de un reforzamiento negativo para evitar el síndrome de abstinencia y un estado emocional desagradable (ansiedad, depresión y/o malestar) (Rodríguez, Gallego, Rodríguez y López, 2012).

El uso abusivo de las tecnologías demanda la necesidad de prestar atención a los procesos cognitivos involucrados en tal adicción y a los rasgos de la personalidad que son vulnerables y tienden a convertir el beneficio, accesibilidad, disponibilidad y disfrute de las diferentes tecnologías a las que tienen acceso las personas, en una conducta adictiva que repercute negativamente en la vida diaria del sujeto que padece tal adicción, reflejándose a su vez en el estado afectivo del sujeto, que sin duda tendrá una clara repercusión en su calidad de vida. En este sentido, se ha relacionado la baja autoestima con sujetos adictos a sustancias; por lo tanto, al considerar el uso abusivo del móvil como una adicción, debería compartir con la adicción a sustancias rasgos psicopatológicos homólogos (Pedrero-Pérez et al., 2012). Los estudios realizados sobre la adicción a las tecnologías han puesto de manifiesto ciertos rasgos de personalidad y estados emocionales que indican una mayor fragilidad anímica y predisposición a la adicción tales como: la impulsividad, la disforia, intolerancia a estímulos no apetitivos (dolores, preocupaciones, disgustos) y excesiva búsqueda de emociones fuertes (Olivencia-Carrión, Pérez-Marfil, Ramos-Revelles, y López-Torrecillas, 2016; Cía, 2018). También se ha hecho referencia a la posibilidad de que la adicción venga desencadenada o sea consecuencia de problemas de personalidad como ser extremadamente tímido o no aceptarse físicamente, así como presentar un estilo de afrontamiento inapropiado, en especial cuando la personalidad se está formando, como se ha evidenciado en la relación encontrada entre personalidad y consumo de sustancias psicoactivas en estudiantes con edades comprendidas entre los 13 y los 18 años (Gonzálvez, Espada, Guillén-Riquelme y Secades, 2016). Todos estos datos apuntan a una compleja relación entre personalidad y frecuencia de uso tecnológico (véase Hussain y Pontes, 2018).

En este sentido, la mayoría de los autores coinciden en considerar que un deficitario autocontrol, junto a otras variables como asertividad, estilo atribucional y autoeficacia (e.g., López-Torrecillas, Peralta, Muñoz-Rivas y Godoy, 2003), serían criterios centrales y comunes tanto en las adicciones con sustancia como en las adicciones de tipo comportamental, lo que deriva en el consumo o uso impulsivo del elemento adictivo y en la incapacidad de posponer la gratificación inmediata que se genera tras su consumo (bien en la primera fase, cuando el elemento adictivo es un reforzador positivo o bien en la fase final cuando se ha convertido en reforzador negativo y es necesario el consumo o uso para eliminar las conductas desagradables tipo ansiedad, depresión, irritabilidad, inquietud, etc. derivadas del síndrome de abstinencia).

Se han encontrado evidencias sobre el uso problemático del dispositivo móvil, alcanzando cifras de hasta un 38\% (más de 6 horas los fines de semanas) y un 37\% el ordenador (más de 3 horas los fines de semana) (Gairín Sallán, y Mercader, 2018) considerando un uso excesivo de estas tecnologías cuando se emplean más de tres horas al día, moderado cuando se dedican entre una y dos horas, y una baja frecuencia de uso cuando se emplea menos de una hora diaria (Pedrero-Pérez et al., 2012; Secades-Villa et al., 2014; Olivencia-Carrión et al., 2016; Gairín et al., 2018). Siguiendo esta dirección, el presente trabajo tiene como objetivo principal conocer la relación existente entre las dimensiones de personalidad y estado afectivo con las principales variables neuropsicológicas involucradas en una conducta adictiva (atención, control inhibitorio/impulsividad) incluyendo la frecuencia de uso como posible criterio diagnóstico, en tanto esta medida sería la más representativa de un posible trastorno adictivo, observando si, en la población de adultos jóvenes, existe un patrón específico de características que favorecerían la adicción a las Nuevas Tecnologías.

Por tanto, las hipótesis de investigación establecidas son las siguientes: La frecuencia y percepción problemática de uso tecnológico en población joven adulta se relaciona de manera inversa con el control inhibitorio $\left(\mathrm{H}_{1}\right)$; La frecuencia y percepción problemática de uso tecnológico en población joven adulta se relaciona de manera inversa con las capacidades en atención selectiva y sostenida $\left(\mathrm{H}_{2}\right)$; La frecuencia y percepción problemática de uso tecnológico en población joven adulta se relaciona de manera directa con los niveles de neuroticismo e introversión $\left(\mathrm{H}_{3}\right)$; La frecuencia y percepción problemática de uso tecnológico en población joven adulta se relaciona de manera directa e inversa con los niveles de afecto negativo y positivo, respectivamente $\left(\mathrm{H}_{4}\right)$. 


\section{Método}

\section{Participantes}

La muestra estuvo compuesta por 90 sujetos españoles $(53,3 \%$ mujeres) con edades comprendidas entre 18 y 34 años $(M=24,11$; $D T=2,78)$. Los participantes fueron alumnos de Máster de una universidad privada, estudiantes y graduados en diferentes carreras universitarias públicas y privadas, conocedores del objetivo del estudio a través de un anuncio previo por redes sociales. Fueron informados previamente al inicio de la evaluación de los objetivos que tiene la investigación, así como de la posibilidad de retirarse del estudio en cualquier momento mediante un consentimiento informado a firmar antes del comienzo de la prueba.

\section{Instrumentos}

Test de Stroop. Test diseñado para valorar la capacidad del examinado para inhibir respuestas automáticas. La prueba está compuesta de tres tareas que han de pasarse en el siguiente orden: 1) Lectura de palabras; 2) Denominación de colores; 3) Interferencia palabra-color. Permite calcular el nivel de interferencia, controlar la tendencia automática a leer palabras y la inhibición de respuesta automática. Los aspectos que evalúa principalmente son: control inhibitorio, atención selectiva visual y velocidad de procesamiento. Cada una de las tres partes que compone el test está formada por 100 elementos por página (distribuidos en 5 columnas con 20 elementos cada una) tres colores (rojo, verde y azul) y XXX coloreadas como símbolo en la evaluación de colores. Los elementos que componen la página número uno son el nombre de los tres colores empleados en el test, repetidos de forma aleatoria y de color negro. La página número dos está compuesta por cinco columnas de ' $X X X$ ', coloreadas de manera aleatoria con los tres colores empleados en el test. Finalmente, en la página número tres aparece de nuevo el nombre de los tres colores empleados en el test, pero impresos en tinta coloreada, al azar y sin concordancia entre el nombre del color y el color de la tinta en que está impreso (Tirapu-Ustárroz, Muñoz-Céspedes, Pelegrín-Valero, y Albéniz-Ferreras, 2005) y la tarea consiste en denominar el color en que está escrita la palabra. El control de la interferencia se obtiene mediante la diferencia entre el total de colores denominados en la tercera parte del test (PC), rendimiento real, y el rendimiento estimado $\left(P C^{\prime} ; P C^{\prime}=[(P x C) /(P+C)]\right)$, fórmula elaborada por Golden (Cabañas y Korzeniowski, 2015). Además, posee buena propiedad psicométrica al presentar una alta consistencia interna $(\alpha=0,89$; Golden, 2005) y está validado con población de 6-85 años.

Trail Making Test (TMT, test de senderos). Consta de dos partes, A y B, con un ensayo de prueba previo a la realización de cada parte. En la parte $A$, el sujeto debe unir círculos numerados y distribuidos al azar sobre la hoja, según el orden numérico correcto $(1,2,3$, 4, etc.). En la segunda parte (forma $\mathrm{B})$, además de círculos con números, se agregan círculos con letras. Se le pide al sujeto que una los números en orden ascendente y las letras en orden alfabético, alternando números y letras (1A-2B-3C, etc.), con la restricción de no poder levantar el lápiz del papel hasta que finalice la prueba (Mateo, 2010). Además de valorar la velocidad de procesamiento del sujeto mediante la primera parte del test, también permite medir la flexibilidad cognitiva, porque se debe inhibir una tarea automática (la secuencia de cifras) que había ya practicado en la parte A, para alternar con una secuencia de letras (parte B). En general, el sujeto impulsivo comete numerosos errores, mientras que el inatento demora más tiempo en la ejecución de la prueba. Está validada por una muestra de 911 participantes, sin deterioro cognitivo, con edades comprendidas entre los 18 y 89 años (Tombaugh, 2004; Tamayo, Casals-Coll, Sánchez-Benavides, Quintana, Manero, Rognoni, Calvo, Palomo, Aranciva, y Peña-Casanova, 2012), con una fiabilidad test-retest que fluctúa entre 0,60 y 0,90 (Spreen y Strauss, 1998; Filippetti, Krumm, y Raimondi, 2015).

Test de Atención d2. Prueba que permite medir la capacidad de atención selectiva y concentración del sujeto evaluado a través de la valoración de la velocidad de procesamiento, precisión y control atencional. Al ser una tarea que dura más de tres minutos también se considera una prueba de atención sostenida, puesto que el sujeto debe señalar con una línea los estímulos diana, pudiendo corregir los errores cometidos (comisiones). Consta de 14 líneas similares compuestas por 47 caracteres, formados tanto por estímulos objetivos (letras "d" con dos rayitas) como distractores (letras "d" o "p" acompañadas de más de dos o menos de dos rayitas) dispuestos aleatoriamente, y por una línea de entrenamiento, para que el sujeto se familiarice con la prueba y se asegure su correcta comprensión, previa a la ejecución del test. La tarea del evaluado, por tanto, consiste en revisar el contenido de cada 
línea, de izquierda a derecha, y marcar aquellas letras "d" que estén acompañadas de dos rayitas pequeñas (dos rayitas arriba, dos abajo, o bien una arriba y otra abajo). Para la compleción de cada línea el sujeto dispone de 20 segundos.

Las puntuaciones que pueden sacarse a partir de este test son las siguientes: TR: medida de velocidad de procesamiento y cantidad de trabajo realizado; TA o total de aciertos: medida de la precisión del procesamiento; Errores: cometidos hasta la última marca hecha en cada fila de elementos. Se pueden cometer diferentes errores: a) Omisión: aquellos estímulos objetivos que no se han marcado; b) Comisión: número de estímulos distractores que han sido marcados. Supone una medida de la precisión del procesamiento y del control inhibitorio; Puntuaciones Totales: 1) TOT: TR- $(\mathrm{O}+\mathrm{C})$. Permite conocer la efectividad que ha tenido el sujeto en la prueba; 2) $\mathrm{CON}=\mathrm{TA}-\mathrm{C}$. Supone la medida de la capacidad de concentración que posee el evaluado; 3$)$ VAR= (TR+)-(TR-). Es la puntuación que se obtiene de la diferencia de la línea que posee el mayor número de caracteres intentados con respecto a la línea que tiene el menor número de elementos intentados. Cuenta con baremos de población general de 8-18 años y de adultos de 19-88 años y con unas buenas propiedades psicométricas $(r>0,90)$ (Brickenkamp y Cubero, 2002).

Cuestionario de Uso Problemático de Nuevas Tecnologías (UPNT). Cuestionario compuesto por 6 partes que permite medir la frecuencia de uso de las tecnologías y detectar un posible uso problemático/excesivo de las mismas. Está validado con una muestra de 2747 estudiantes (de $5^{\circ}$ de primaria a $5^{\circ}$ de licenciatura, de 9-33 años). Está formado por un total de 41 ítems, siendo los dos primeros ítems los dedicados a estimar la frecuencia y problemas en el uso de las diferentes tecnologías. El resto está agrupado en 4 categorías diferentes permitiendo conocer la frecuencia de uso de Internet, Video-juegos y Móvil mediante 10 ítems (en cada clasificación), y los 9 restantes proporcionan información sobre el uso de la Televisión. El primer apartado de las preguntas 3, 4, 5 y 6 se refiere al lugar donde se usan los dispositivos, el segundo apartado hace referencia al total de horas semanales de uso (excepto el ítem 5.2 donde se pregunta por la persona que corre con los gastos del teléfono móvil) y el tercero al total de horas diarias. En el caso del apartado 5, los ítems 5.3 y 5.4 son los dedicados a señalar el tiempo de uso semanal y diario respectivamente. El resto de ítems (del 4 al 10 en los apartados 3 y 4 ; del 5 al 10 en el apartado 5; y del 4 al 9 en el apartado 6) permite medir la percepción que posee la persona respecto al uso que realiza de las tecnologías evaluadas con un modelo de respuesta de Nunca, A veces, Con frecuencia o Siempre.

La fiabilidad del cuestionario muestra un alfa de Cronbach adecuado, en concreto la escala total $(0,876)$, correspondiendo la fiabilidad más baja a la subescala de Televisión, aunque en todos los apartados se supera el criterio establecido $(0,70)$. A su vez, el cuestionario muestra una alta correlación de las distintas subescalas con la escala total (superiores a 0,66$)$ y una alta significación $(p<0,001)$, obteniendo una correlación entre subescalas con valores medios (entre 0,35 y 0,55), a excepción de los apartados Videojuegos y Teléfono Móvil $(0,18)$, aunque todas son significativas $(p<0,001)$. Además, cuenta con una adecuada validez clínica al obtener diferencias significativas en las diferentes escalas y niveles de significación superiores a 0,001, evaluada a través de la comparación entre el grupo que considera que posee problemas de uso de las tecnologías y el grupo que no (Labrador, Villadangos, Crespo y Becoña, 2013).

Cuestionario de Personalidad (EPQR-A). Versión española del cuestionario EPQ validado con una nuestra de 18-39 años (DT mujeres $=6,3$, edad media 21,5; DT hombres $=7,1$, edad media 21,1). Está compuesto de 24 ítems para evaluar 4 subescalas (3 dimensiones de la personalidad: Extraversión, Neuroticismo y Psicoticismo; y una subescala de la tendencia a mentir o emitir respuestas de deseabilidad social). Cada subescala está formada por 6 ítems con un formato de respuesta Sí (1) vs No (0).

La fiabilidad del cuestionario, calculada mediante los coeficientes de alfa de Cronbach, muestra niveles entre buenos y aceptables para las dimensiones de Extraversión $(0,74)$ y Neuroticismo $(0,78)$ y admisibles para las subescalas de Psicoticismo $(0,63)$ y Sinceridad $(0,54)$. La validez convergente y divergente han sido obtenidas mediante la correlación de Pearson entre las subescalas del EPQR-A con las subescalas del EPQ, PANAS, rasgo de ansiedad, sensibilidad a la ansiedad y preocupación, alcanzando una validez aceptable, principalmente en las subescalas de Extraversión y Neuroticismo. Por tanto, el EPQR-A posee una adecuada validez convergente y divergente ya que el Neuroticismo presenta una elevada correlación con el afecto negativo del PANAS $(0,57)$, con el rasgo de ansiedad del Cuestionario de Ansiedad Estado-Rasgo STAI $(0,69)$, con la preocupación del Cuestionario de Pre- 
ocupación PSWQ $(0,66)$ y con la misma dimensión del EPQ $(0,79)$; la Extraversión correlaciona con el afecto positivo del PANAS $(0,57)$ y con el EPQ $(0,86)$; $y$, aunque en menor medida que las anteriores dimensiones, el Psicoticismo y la Sinceridad también muestran correlaciones con la misma dimensión del EPQ (Sandín, Valiente, Montes, Chorot y Germán, 2002).

Cuestionario de Afecto positivo y negativo (PANAS). Instrumento autoadministrado que permite estimar el estado afectivo de un sujeto a partir de una lista de adjetivos que describen diferentes emociones. Consta de 20 ítems, 10 referidos al afecto positivo (ítems impares) y 10 al afecto negativo (ítems pares). Cada ítem se puntúa según una escala tipo Likert con 5 opciones de respuesta: 1 «Nada»; 2 «Un poco», 3 «Moderadamente», 4 «Bastante», 5 «Mucho» puntuando cada ítem según como se haya sentido en el último mes. La escala que obtenga mayor puntuación con respecto a la otra es la que indica la prevalencia de las emociones positivas frente a las negativas en el sujeto o viceversa (Blanco Molina y Salazar Villanea, 2014). La prueba presenta un coeficiente alfa de Cronbach de 0,92 para AP (afecto positivo) y de 0,88 para AN (afecto negativo), siendo mayores que los índices obtenidos por los autores de la prueba original (Watson et al., 1988). A su vez, está validada con población española por tres muestras diferentes, la primera compuesta por un rango de 16-60 años, la segunda por estudiantes universitarios con edades comprendidas entre los 19-58 años, y la tercera por una muestra clínica de mujeres con trastorno depresivo mayor o distimia (de 26-83 años) (López-Gómez, Hervás y Vázquez, 2015).

\section{Procedimiento}

La batería de pruebas fue administrada en el mismo orden y de forma individual para cada uno de los participantes del estudio, asegurando el anonimato y confidencialidad de los datos mediante la asignación de un número específico para cada uno de ellos. En primer lugar, tras la lectura del consentimiento y explicación de las posibles dudas de los participantes, se comenzaba el estudio aplicando las dos tareas seleccionadas para medir el control inhibitorio, primero el Stroop, para evaluar la capacidad de control inhibitorio verbal, seguida del Trail Making Test, para conocer la inhibición motora. Una vez completadas ambas tareas, se proseguía con la prueba psicométrica destinada a medir la capacidad atencional, el Test de Atención d2. El motivo de administrar primero las pruebas que valoran el control inhibitorio frente a la prueba atencional se debe a la importancia que presentan los procesos cognitivos que mide cada prueba en la adicción comportamental (presente en todas las investigaciones revisadas el deficitario control de impulsos en un sujeto con adicción frente a las hipótesis establecidas sobre un posible descenso de la capacidad atencional en sujetos con tal característica). A continuación, se procede a la cumplimentación de los cuestionarios auto-administrados seleccionados para valorar la frecuencia de uso de las tecnologías (UPNT), la personalidad (EPQR-A) y el estado afectivo (PANAS). En este caso, el orden de administración de los cuestionarios se debe tanto a la longitud de los mismos (considerando la posible aparición de fatiga y reducción de concentración en la fase final de la valoración) como a la importancia que posee cada uno de ellos en el estudio, siendo el Cuestionario de Uso Problemático de las Nuevas Tecnologías (UPNT) la prueba central de la investigación, y considerada como posible herramienta diagnóstica para la adicción a las tecnologías. Respecto a esta prueba, se pidió a los participantes que indicaran la frecuencia de uso de cada uno de los dispositivos para el entretenimiento, sin tener en cuenta su uso para el trabajo o los estudios.

\section{Análisis de datos}

Para analizar las relaciones existentes entre las variables neuropsicológicas, personalidad y estado afectivo con la percepción problemática y frecuencia de uso de las tecnologías se ha utilizado la correlación de Pearson. Los análisis ejecutados se han llevado a cabo con el programa estadístico SPSS15.0. 


\section{Resultados}

Previamente a los análisis presentados, se examinaron los datos para identificar casos perdidos, atípicos y el cumplimiento de los supuestos de normalidad. No se identificaron casos perdidos ni atípicos y se ha cumplido del supuesto de normalidad (a través de los índices de asimetría, curtosis y Kolmogorov-Smirnov).

\section{Análisis descriptivo}

Tabla 1

Análisis descriptivo de las pruebas neuropsicológicas, personalidad y afecto.

\begin{tabular}{|c|c|c|c|}
\hline & M & DT & Min-Max \\
\hline Stroop_P_PD & 119,54 & 12,06 & $92-150$ \\
\hline Stroop_C_PD & 81,74 & 11,45 & $48-113$ \\
\hline Stroop_PC_PD & 53,81 & 10,41 & $32-76$ \\
\hline Stroop_PC'PD & 48,36 & 5,38 & $32,60-63,10$ \\
\hline Stroop_INTERF_PD & 5,51 & 7,87 & $-16,90-25,60$ \\
\hline TMTA_Tiempo & 25,80 & 8,08 & $13-54,00$ \\
\hline TMTB_Tiempo & 59,71 & 24,45 & $20-148$ \\
\hline d2_PcTR & 60,90 & 26,37 & $10-99$ \\
\hline d2_PcAciertos & 54,24 & 28,81 & $2-99$ \\
\hline d2_PcOmisiones & 39,04 & 30,80 & $1-99$ \\
\hline d2_PcComisiones & 18,67 & 22,34 & 1,99 \\
\hline d2_РcTOT & 58,58 & 26,82 & $2-99$ \\
\hline d2_PcCON & 54,42 & 28,12 & $1-99$ \\
\hline Neuroticismo & 2,89 & 1,70 & $0-6$ \\
\hline Extraversión & 4,13 & 0,78 & $2-6$ \\
\hline Psicoticismo & 3,49 & 1,00 & $1-6$ \\
\hline Sinceridad & 3,38 & 1,42 & $1-6$ \\
\hline Afecto_Positivo & 35,23 & 5,95 & $20-48$ \\
\hline Afecto_Negativo & 23,38 & 7,92 & $10-42$ \\
\hline
\end{tabular}

Nota. Stroop Palabras (Stroop P); Stroop Colores (Stroop C); Stroop Palabra-Color (Stroop PC); Stroop rendimiento estimado (Stroop PC'); Trail Making Test parte A (TMTA); Trail Making Test parte B (TMTB); Total de Respuestas en Test d2 (d2 Pc TR); Total de Aciertos en Test d2 (d2 Pc TA); Efectividad en Test d2 (d2 Pc TOT); Concentración en Test d2 (d2 Pc CON); Errores por Omisión en Test d2 (d2 Pc Omisiones); Errores por Comisión en Test d2 (d2 Pc Comisiones).

Relación entre frecuencia y percepción de uso tecnológico y variables neuropsicológicas.

Los resultados mostraron una relación significativa entre el uso de Internet y la interferencia real palabra-color $(r=-0,236)$ y el Total de Aciertos en la tarea atencional $(r=-0,248)$, evidenciándose que los sujetos que más utilizan Internet tienen un peor control inhibitorio verbal y una menor capacidad en atención selectiva y sostenida. En segundo lugar, se observó que la frecuencia de uso de la televisión se relaciona de manera directa con el número de errores por comisión en la prueba de atención $(r=$ 0,252 ). Este resultado estaría evidenciando que aquellos sujetos que más horas dedican a ver la Televisión al día presentan mayor número de estímulos distractores marcados, lo que supone un peor control inhibitorio motor en tareas atencionales. Por último, se identificó una relación significativa e inversa entre la percepción problemática y la frecuencia de uso tecnológico con el Stroop Palabras $(r=-0,242)$ y el rendimiento estimado en el Stroop $(r=-0,248)$, así como con la cantidad de aciertos en la tarea de atención $(r=-0,226)$; es decir, la frecuencia de uso tecnológico estaría relacionada con un descenso de la velocidad de procesamiento en tareas de lectura y un menor control inhibitorio verbal, así como con un deterioro en el rendimiento en tareas atencionales no vinculadas con las actividades tecnológicas (véase tabla 2). 
Tabla 2

Análisis de la relación entre frecuencia de uso tecnológico (FUT), percepción problemática (PP) y variables neuropsicológicas.

\begin{tabular}{|c|c|c|c|c|c|}
\hline & $\begin{array}{c}\text { FUT } \\
\text { Internet }\end{array}$ & $\begin{array}{c}\text { FUT } \\
\text { Videojuegos }\end{array}$ & $\begin{array}{l}\text { FUT } \\
\text { Móvil }\end{array}$ & $\begin{array}{c}\text { FUT } \\
\text { Televisión }\end{array}$ & PP \\
\hline Stroop_P_PD &,- 094 & ,020 &,- 093 & , 044 &,$- 242^{*}$ \\
\hline Stroop_C_PD &,- 179 &, 063 &,- 160 & ,154 &,- 206 \\
\hline Stroop_PC_PD &,$- 236^{*}$ &, 025 &,- 169 & ,175 &,- 084 \\
\hline Stroop_PC'_PD &,- 171 &, 061 &,- 151 & ,134 &,$- 248^{*}$ \\
\hline Stroop_INTERF_PD &,- 186 &,- 003 &,- 111 & 137 & ,057 \\
\hline TMTA_Tiempo & ,049 &,- 018 & 108 &,- 137 & ,021 \\
\hline TMTB_Tiempo & ,038 & ,098 &, 046 &,- 161 & ,117 \\
\hline d2_PcTR &,- 118 &,- 027 &,- 115 & ,027 &,- 100 \\
\hline d2_PcAciertos &,$- 248^{*}$ &, 002 &,- 186 &,- 003 &,$- 226^{*}$ \\
\hline d2_PcOmisiones &,- 167 &, 020 &,- 187 & ,134 &, 061 \\
\hline d2_PcComisiones &,- 060 &,- 046 &,- 016 &, $252^{*}$ & ,057 \\
\hline d2_PcTOT &,- 174 &,- 032 &,- 188 &, 062 &,- 127 \\
\hline d2_PcCON &,- 196 &,- 006 &,- 204 &, 110 &,- 111 \\
\hline
\end{tabular}

Nota. Stroop Palabras (Stroop P); Stroop Colores (Stroop C); Stroop Palabra-Color (Stroop PC); Stroop rendimiento estimado (Stroop PC'); Trail Making Test parte A (TMTA); Trail Making Test parte B (TMTB); Total de Respuestas en Test d2 (d2 Pc TR); Total de Aciertos en Test d2 (d2 Pc TA); Efectividad en Test d2 (d2 Pc TOT); Concentración en Test d2 (d2 Pc CON); Errores por Omisión en Test $d 2$ (d2 Pc Omisiones); Errores por Comisión en Test d2 (d2 Pc Comisiones). * $p<.05$

Análisis relación frecuencia de uso de Tecnologías, personalidad y afecto.

Los análisis mostraron una relación significativa y directa entre la frecuencia de uso de Internet y los niveles de neuroticismo $(r=0,355)$ y afecto negativo $(r=0,319)$; es decir, que los sujetos que con más frecuencia usan Internet muestran un peor control inhibitorio verbal y menor cantidad de aciertos en tareas de atención selectiva y sostenida, así como mayor inestabilidad emocional y prevalencia de emociones negativas. A su vez, la frecuencia de uso del Móvil correlacionó de forma positiva con el Neuroticismo $(r=0,315)$ y el Afecto Negativo $(r=0,364)$, señalando que a mayor frecuencia de uso del dispositivo móvil mayor tendencia a la inestabilidad emocional y sentimientos de angustia, preocupación, irritabilidad, ansiedad, nerviosismo y culpabilidad, entre otros. Finalmente, en la percepción problemática de uso se encontró una relación significativa positiva con el Neuroticismo $(r=0,303)$ y el Afecto Negativo $(r=0,304)$ (véase tabla 3).

Tabla 3

Análisis de la relación entre frecuencia de uso tecnológico (FUT), percepción problemática (PP), afecto y personalidad.

\begin{tabular}{lccccc}
\hline & $\begin{array}{c}\text { FUT } \\
\text { Internet }\end{array}$ & $\begin{array}{c}\text { FUT } \\
\text { Videojuegos }\end{array}$ & $\begin{array}{c}\text { FUT } \\
\text { Móvil }\end{array}$ & $\begin{array}{c}\text { FUT } \\
\text { Televisión }\end{array}$ & PP \\
\hline Neuroticismo &, $355^{*}$ &, 093 &, $315^{*}$ &, 156 &, $303^{*}$ \\
Extraversión &,- 109 &, 059 &,- 057 &,- 011 &, 095 \\
Psicoticismo &, 020 &, 070 &, 003 &, 065 &,- 040 \\
Sinceridad &, 184 &, 181 &, 136 &, 204 &, 047 \\
Afecto Positivo &, 141 &, 064 &, 053 &, 036 &, 005 \\
Afecto Negativo &, $319^{*}$ &,- 001 &, $364^{*}$ &, $304^{*}$ \\
\hline Nota. Cuestionario de Personalidad (EPQR-A); Cuestionario de Afecto positivo y negativo (PANAS).
\end{tabular}

\section{Discusión y conclusiones}

El presente estudio tuvo como objetivo principal investigar la relación existente entre la frecuencia de uso tecnológico en población adulta joven y los procesos cognitivos de control inhibitorio y atención, así como la personalidad y estado afectivo. A partir de los resultados obtenidos, se pueden aceptar las hipótesis 1 y 2 , en tanto se ha evidenciado relación entre la frecuencia y percepción problemática del uso tecnológico y las variables neuropsicológicas analizadas (inhibición y atención). Estos resultados son congruentes con los obtenidos en otras investigaciones, donde se ha informado del efecto negativo de la alta frecuencia de uso de internet en los procesos inhibitorios (Cabañas, 2015), siendo estos datos corroborados por estudios de neuroimagen, donde se observaban diferencias significativas en el funcionamiento de las estructuras relacionadas con los procesos de inhibición en personas adictas a internet (alta frecuencia de uso), respecto a población normal (Darnai et al., 2019). Asimismo, se observó que los sujetos que invierten más horas diarias a ver la Televisión muestran peor control inhibitorio motor en tareas atencionales al presentar mayor número de errores por comisión (se aceptan 
hipótesis 1 y 2). Estos datos serían congruentes con los obtenidos en población infantil, donde se ha asociado el tiempo de exposición a la Televisión y problemas atencionales (Miller et al., 2007), siendo una aportación relevante en la presente investigación, al observarse tal relación en la población adulta joven. Por otro lado, se ha encontrado relación entre la percepción de uso problemático y algunas de las variables relacionadas con los procesos atencionales e inhibitorios, en el mismo sentido que las encontradas con la frecuencia de uso. Esto nos advierte de que los déficits en los procesos cognitivos podrían ser procesados como señales que advierten a los sujetos de posibles problemas con el uso tecnológico, más allá de la percepción que se tenga del tiempo invertido, que como alguna investigación ha mencionado, puede verse afectado en las personas con este tipo de adicciones (véase Turel y Cavagnaro, 2019).

Por otro lado, los resultados informaron de una correlación significativa positiva entre el Neuroticismo /Afecto Negativo y la cantidad de horas diarias dedicadas al uso de Internet y Móvil (se aceptan hipótesis 3 y 4); es decir, aquellos sujetos que hacen un mayor uso tecnológico evidenciaron una mayor tendencia a la inestabilidad emocional y experimentaron en mayor medida sentimientos de angustia, preocupación, irritabilidad y ansiedad. Estos resultados son congruentes con los aportados por diferentes autores (Cía, 2018; Echeburúa, 2010, 2012; Sánchez-Carbonell, 2008; Sánchez-Martinez, 2009; Pedrero-Pérez, 2012; Rodríguez, 2012), quienes refieren que la inestabilidad emocional, la introversión y un estado de ánimo disfórico son características presentes en los sujetos que realizan un uso abusivo o excesivamente frecuente de las tecnologías. Sin embargo, a pesar de haberse documentado la existencia de relaciones significativas negativas entre un deficitario control inhibitorio y el uso de los Videojuegos (Marco-Puche y Chóliz, 2012; Cabañas et al., 2015), en el presente estudio no se obtuvieron resultados significativos entre la frecuencia de uso de los Videojuegos con las variables neuropsicológicas estudiadas, la personalidad y el estado afectivo.

Los datos aportados por el presente trabajo, en su conjunto, evidencian una relación entre la frecuencia de uso tecnológico y las variables neuropsicológicas de control inhibitorio y atención; además de la implicación de variables de personalidad como el neuroticismo y las afectivas, en un sentido que plantea cuestiones a investigar en futuros trabajos. En concreto, el papel modulador de las variables de personalidad y afectivas en el inicio y mantenimiento de las conductas relacionadas con la adicción a las tecnologías. Uno de los problemas a la hora de abordar esta cuestión será la de determinar cuándo se está hablado de una conducta adictiva y cuando no. En este sentido, algunas investigaciones indican que se debería considerar un uso abusivo la dedicación de dos o tres horas diarias (Pedrero-Pérez et al., 2012; Secades-Villa et al., 2014; Cabañas et al., 2015); sin embargo, en la presente investigación, salvando esta cuestión y utilizando una muestra adulta joven sin adicciones diagnosticadas, se puede constatar la existencia de una relación entre la frecuencia de uso y capacidades cognitivas como la inhibición y la atención, que deberían ser más evidentes en población con adicción al uso tecnológico.

Por último, es importante señalar las limitaciones encontradas en la realización del presente estudio. En primer lugar, la muestra utilizada fue no probabilística, los participantes fueron seleccionados en función de la accesibilidad y recursos disponibles por parte de la responsable del diseño y desarrollo de la presente investigación, por lo que la generalización de los resultados a la población joven española se vería comprometida. Del mismo modo, a pesar de haber utilizado los test clásicos empleados para evaluar la capacidad de control inhibitorio verbal y motor (Stroop y TMT, respectivamente), así como la capacidad de atención selectiva y sostenida (Test Atencional d2), es necesario tener en cuenta que éstos valoran de forma simultánea otros procesos cognitivos y no únicamente la función cognitiva que era objeto de estudio. Además, como señala Carbonell (2012), existe un problema en la recogida y medida real de la frecuencia de uso tecnológico, ya que el único método existente hasta la fecha se basa en la compleción de un cuestionario que supone más bien una medida perceptiva y subjetiva de su uso. Por tanto, sería conveniente desarrollar una medida objetiva del uso tecnológico, en sus diferentes modalidades, para emplearla en futuras investigaciones y poder sacar conclusiones con mayor validez y fiabilidad que las desarrolladas hasta la actualidad. Finalmente, sería adecuado incluir en los próximos estudios una medida del uso de las tecnologías en los diferentes ámbitos de la vida cotidiana, permitiendo conocer el tiempo dedicado al uso de Internet, Videojuegos, Móvil y Televisión, así como su promedio, en cada una de las esferas en las que se desenvuelve la población seleccionada para el estudio. Todo esto permitiría, el diseño de nuevas estrategias y técnicas de prevención e intervención que perfeccionen, actualicen y complementen a las terapias psicoeducativas presentes en la actualidad, y, por otro lado, seguir avanzando en la comprensión de los beneficios y perjuicios que pueden desarrollarse con el uso continuado de las tecnologías. 


\section{Referencias}

1. American Psychiatric Association. (2000). Diagnostic and statistical manual of mental disorders (4th ed). Washington, DC: Author.

2. American Psychiatric Association. (2014).Guía de consulta de los criterios diagnósticos del DSM$5 \circledR$ : Spanish edition of the desk reference to the diagnostic criteria from $D S M-5 \AA$. American Psychiatric Pub. https://doi.org/10.1176/appi.books.9780890425657

3. Andreassen, C. S., Billieux, J., Griffiths, M. D., Kuss, D. J., Demetrovics, Z., Mazzoni, E., y Pallesen, S. (2017). Adicción a internet, redes sociales y videojuegos: La relación entre el uso adictivo de las redes sociales y los video juegos y síntomas de trastornos psiquiátricos: un estudio transversal a gran escala. RET: Revista de Toxicomanías, 81, 2-2.

4. Arango-Forero, G., Bringué-Sala, X., y Sádaba-Chalezquer, C. (2010). La generación interactiva en Colombia: adolescentes frente a la Internet, el celular y los videojuegos. Anagramas, 9, 45-56. https://doi.org/10.22395/angr.v9n17a3

5. Billieux, J., Philippot, P., Schmid, C., Maurage, P., De Mol, J., y Van der Linden, M. (2015). Is dysfunctional use of mobile phone a behavioural addiction? Confronting symptom-based versus process-based approaches. Clinical Psychology and Psychotherapy, 22, 460-468. https://doi. org/10.1002/cpp.1910

6. Blanco-Molina, M., y Salazar-Villanea, M. (2014). Escala de Afectividad (PANAS) de Watson y Clark en Adultos Mayores. En V. Smith-Castro (Comp.), Compendio de Instrumentos de Medición IIP-2014. http://iip.ucr.ac.cr/sites/default/files/cuadernosmetodologicos/cuamet6.PDF

7. Brickenkamp, R., y Cubero, N. S. (2002). D2: test de atención. Madrid: Tea.

8. Britos, E. M., y Brítez, R. (2015). Nuevas Tecnologías y Juventud: Uso y Acceso a Internet, Móvil y Redes Sociales en universitarios de ambos sexos, de una Carrera de Humanidades (Psicología), de una Universidad Privada, período 2014. Revista Científica de la UCSA, 2, 63-74. https:// doi.org/10.18004/ucsa/2409-8752/2015.002(02)063-074

9. Cabañas, M., y Korzeniowsk, C. (2015). Uso de celular e internet: su relación con planificación y control de la interferencia. Revista Argentina de Ciencias del Comportamiento (RACC), 7, 3.

10. Carbonell, X., Fuster, H., Chamarro, A., y Oberts, U. (2012). Adicción a Internet y móvil: una revisión de estudios empíricos españoles. Papeles del psicólogo, 33, 82-89.

11. Carbonell, X., Chamarro, A., Griffith M., Oberst U., Cladellas, R., y Talarn, A. (2012). Problematic Internet and cell phone use in Spanish teenagers and young students. Anales de psicología, 28, 789-796. https://doi.org/10.6018/analesps.28.3.156061

12. Castro, J. A., Vinaccia, S., y Ballester-Arnal, R. (2018). Ansiedad social, adicción al internet y al cibersexo: su relación con la percepción de salud. Terapia psicológica, 36, 134-143. https://doi. org/10.4067/S0718-48082018000300134

13. Chóliz, M. (2010). Guía PrevTec 3.1 Programa de Prevención de Adicciones Tecnológicas. Valencia: FEPAD.

14. Cía, A. H. (2018). Las adicciones no relacionadas a sustancias (DSM-5, APA, 2013): un primer paso hacia la inclusión de las Adicciones Conductuales en las clasificaciones categoriales vigentes. Inmanencia. Revista del Hospital Interzonal General de Agudos (HIGA) Eva Perón, 6(1).

15. Darnai, G., Perlaki, G., Zsidó, A. N., Inhóf, O., Orsi, G., Horváth, R., Nagy, S. A., Lábadi, B., Tényi, D., Kovács, N., Dóczi, T., Demetrovics, Z., y Janszky, J. (2019). Internet addiction and functional brain networks: task-related fMRI study. Scientific Reports, 9, 15777. https://doi.org/10.1038/ s41598-019-52296-1

16. Echeburúa, E. (1999). ¿Adicciones... sin drogas? Las nuevas adicciones (juego, sexo, comida, compras, trabajo, Internet). Bilbao. Desclée de Brouwer.

17. Echeburúa, E., Corral, P., y Amor, P.J. (2005). El reto de las nuevas adicciones: objetivos terapéuticos y vías de intervención. Psicología Conductual, 13, 511-525.

18. Echeburúa, E., y Corral, P. (2010). Adicción a las nuevas tecnologías y a las redes sociales en jóvenes: un nuevo reto. Adicciones, 22, 91-96. https://doi.org/10.20882/adicciones.196

19. Echeburúa, E. y Requesens, A. (2012). Adicción a las redes sociales y a las nuevas tecnologías en jóvenes y adolescentes. Guía para educadores. Madrid: Pirámide.

20. Filippetti, V. A., Krumm, G., y Raimondi, W. (2015). Funciones Ejecutivas y sus correlatos con Inteligencia Cristalizada y Fluida: Un estudio en Niños y Adolescentes. Neuropsicología Latinoamericana, 7, 24-33.

21. Gairín Sallán, J., y Mercader, C. (2018). Usos y abusos de las TIC en los adolescentes. Revista De Investigación Educativa, 36, 125-140. https://doi.org/10.6018/rie.36.1.284001

22. Golden, C. (1978). Test de colores y palabras de Stroop. Madrid. TEA. 
23. Golden, C.J. (2005). Stroop Test de colores y palabras. Manual (4a edición). Madrid: TEA.

24. Gonzálvez, M. T., Espada, J. P., Guillén-Riquelme, A. y Secades, R. (2016). Asociación entre rasgos de personalidad y consumo de sustancias en adolescentes españoles. Adicciones, 28, 108-115.

25. Hussain, Z., y Pontes, H. M. (2018). Personality, internet addiction, and other technological addictions: A psychological examination of personality traits and technological addictions. In B. Bozoglan (Ed.), Psychological, social, and cultural aspects of Internet addiction (pp. 45-71). Hershey, PA: IGI Global. https://doi.org/10.4018/978-1-5225-3477-8.ch003

26. Instituto Nacional de Estadística (2017). Encuesta sobre equipamiento y uso de tecnologías de información y comunicación en los hogares. Disponible en http://www.ine.es/prensa/tich_2017. pdf. Accedido 2 de febrero 2018.

27. Instituto Nacional de Estadística (2019). Encuesta sobre equipamiento y uso de tecnologías de información y comunicación en los hogares.

28. Labrador, F. J., y Villadangos, S. M. (2010). Menores y nuevas tecnologías: conductas indicadoras de posible problema de adicción. Psicothema, 22, 180-188.

29. Labrador, F. J., Villadangos, S. M., Crespo, M., y Becoña, E. (2013). Desarrollo y validación del cuestionario de uso problemático de nuevas tecnologías (UPNT). Anales de psicología, 29, 836847. https://doi.org/10.6018/analesps.29.3.159291

30. Leung, L. (2007). Leisure boredom, sensation seeking, self-esteem, addiction symptoms and patterns of cell phone use. En E. A. Konijn, M. A. Tanis, S. Utz y A. Linden (eds.), Mediated interpersonal communication (pp. 359-381). Mahwah, NJ: Lawrence Erlbaum Associates.

31. López-Gómez, I., Hervás, G., y Vázquez, C. (2015). Adaptación de la "Escala de afecto positivo y negativo" (PANAS) en una muestra general española. Psicología conductual, 23, 529-548.

32. López-Torrecillas, F., Peralta, I., Muñoz-Rivas, M J. y Godoy, J. F. (2003). Autocontrol y consumo de drogas. Adicciónes, 15(2), 127-136. https://doi.org/10.20882/adicciones.436

33. Miller, C.J., Marks, D. J., Miller, S. R., Berwid, O. G., Kera, E. C., Santra, A., y Halperin, J. M. (2007). Television viewing and risk for attention problems in preschool children. Journal of Pediatric Psychology, 32, 448-452. https://doi.org/10.1093/jpepsy/jsl035

34. Marks, I. (1990). Behavioural (non-chemical) addictions. British Journal of Addictions, 85, 13891394.

35. Marco-Puche, C. y Chóliz, M. (2012). Impulsividad y dependencia de videojuegos en adolescentes. En Gonzalez, C.; Gonzalez, D; Guil, R. y Mestre, J. Aportaciones recientes al estudio de la motivación y las emociones. Sevilla: Fenix.

36. Mateo, V. F. (2010). Neuropsicología Infantil: Intento de validación del Trail-Making Test en población escolar no patológica. Quaderns Digitals: Revista de Nuevas Tecnologías y Sociedad, 65, 17.

37. Odriozola, E. E. (2012). Factores de riesgo y factores de protección en la adicción a las nuevas tecnologías y redes sociales en jóvenes y adolescentes. Revista Española de Drogodependia, 4, 435-48.

38. Olivencia-Carrión, M., Pérez-Marfil, M., Ramos-Revelles, M., y López-Torrecillas, F. (2016). Personalidad y su relación con el uso versus abuso del teléfono móvil. Acción psicológica, 13, 109-118. https://doi.org/10.5944/ap.13.1.17427

39. Pedrero-Pérez, E., Rodríguez-Monje, M. T., y Ruiz-Sánchez de León, J. M. (2012). Adicción o abuso del teléfono móvil. Revisión de la literatura. Adicciones, 24, 139-152. https://doi. org/10.20882/adicciones.107

40. Rial, A., Gómez, P., Braña, T., y Varela, J. (2014). Actitudes, percepciones y uso de Internet y las redes sociales entre los adolescentes de la comunidad gallega (España). Anales de psicología, 30(2), 642-655. https://doi.org/10.6018/analesps.30.2.159111

41. Rodríguez, O., Gallego, V., Rodríguez, M. J., y López, M. Á. (2012). Adicción a las nuevas tecnologías. Psicología de las Adicciones, 1, 2-6.

42. Sánchez-Carbonell, X., Beranuy, M., Castellana, M., Chamarro, A., y Oberst, U. (2008). La adicción a Internet y al móvil:¿ moda o trastorno?. Adicciones, 20. https://doi.org/10.20882/adicciones. 279

43. Sánchez-Martínez, M., y Otero, A. (2009). Factors associated with cell phone use in adolescents in the community of Madrid (Spain). Cyberpsychology and Behavior, 12, 131-137. https://doi. org/10.1089/cpb.2008.0164 
44. Sandín, B., Valiente, R. M., Montes, M. O., Chorot, P., y Germán, M. A. S. (2002). Versión española del cuestionario EPQR-Abreviado (EPQR-A)(II): Replicación factorial, fiabilidad y validez. Revista de psicopatología y psicología clínica, 7, 207-216. https://doi.org/10.5944/rppc. vol.7.num.3.2002.3934

45. Secades-Villa, R., Calafat, A., Fernández-Hermida, J.R., Juan, M., Duch, M., Skärstrand, E. Becoña, E., y Talic, S. (2014). Duration of Internet use and adverse psychosocial effects among European adolescents. Adicciones, 26, 247-253. https://doi.org/10.20882/adicciones.6

46. Spreen, O., y Strauss, E. (1998). A Compendium of Neuropsychological Test: Administration, Norms and Commentary (2nd. ed.). New York: Oxford University Press.

47. Tamayo, F., Casals-Coll, M., Sánchez-Benavides, G., Quintana, M., Manero, R. M., Rognoni, T., Calvo, L., Palomo, R., Aranciva, F., y Peña-Casanova, J. (2012). Estudios normativos españoles en población adulta joven (Proyecto NEURONORMA jóvenes): normas para las pruebas span verbal, span visuoespacial, Letter-Number Sequencing, Trail Making Test y Symbol Digit Modalities Test. Neurología, 27, 319-329. https://doi.org/10.1016/j.nrl.2011.12.020

48. Tombaugh, T. N. (2004). Trail Making Test A and B: normative data stratified by age and education. Archives of clinical neuropsychology, 19, 203-214. https://doi.org/10.1016/S08876177(03)00039-8

49. Treuer, F., Fabian, Z. y Furedi, J. (2001). Internet addiction associated with features of impulse control-disorder: is it a real psychiatric disorder? Journal of Affective Disorders, 66, 283. https:// doi.org/10.1016/S0165-0327(00)00261-5

50. Tirapu-Ustárroz, J., Muñoz-Céspedes, J. M., Pelegrín-Valero, C., y Albéniz-Ferreras, A. (2005). Propuesta de un protocolo para la evaluación de las funciones ejecutivas. Revista de neurología, 41, 177-186. https://doi.org/10.33588/rn.4103.2005054

51. Turel, O., y Cavagnaro, D. (2019). Effect of Abstinence from Social Media on Time Perception: Differences between Low- and At-Risk for Social Media "Addiction" Groups. Psychiatry Quarterly, 90, 217-227. https://doi.org/10.1007/s11126-018-9614-3

52. Van Rooij, A. y Prause, N. (2014). A critical review of "Internet addiction" criteria with suggestions for the future. Journal of Behavioral Addictions, 3, 203-213. https://doi.org/10.1556/JBA.3.2014.4.1

53. Yudes, C., Rey, L., y Extremera, N. (2019). Ciberagresión, adicción a internet e inteligencia emocional en adolescentes: un análisis de diferencias de género. Voces de la Educación, 27-44.

RECIBIDO: 23 de septiembre de 2019 MODIFICADO: 7 de febrero de 2020 ACEPTADO: 26 de junio de 2020 